\title{
Enhanced Hairpin Stability through Loop Design: The Case of the Protein G B1 Hairpin
}

R. Matthew Fesinmeyer, F. Michael Hudson, and Niels H. Andersen,*

Department of Chemistry, University of Washington, Seattle WA 98195

\section{SUPPORTING INFORMATION}

Loop Mutation Selection Based on Residue Occurrence Statistics in Protein Hairpins - We employed the SLoops database[www-cryst.bioc.cam.ac.uk/ sloop] $]^{1}$; to determine the residue statistics for $\mathbf{E}-\mathrm{E}-\alpha_{R}-\alpha_{R}-\gamma_{R}-\alpha_{L}-E-\mathbf{E}(n=223)$ and the related $\mathbf{E}-b-\alpha_{R}-\alpha_{R}-\gamma_{R}-\alpha_{L}-b-E(n=62)$ loops. The results for the 12 residues that display a preference for loop positions rather than strand locations appear below. For each hairpin type, the fractional occurrence of each residue at the eight central residues is shown. The largest value ( 0.66 for Gly in T4 position of $\mathbf{E}-b-\alpha_{R}-\alpha_{R}-\gamma_{R}-\alpha_{L}-b-\mathbf{E}$ hairpins indicates that $66 \%$ of these hairpins have a glycine at that position. In addition, we calculated the propensity of each residue to occur at a specific loop position; propensity values of 1.0 would indicate equal fractions at all loop positions. With the exception of His, which is poorly represented in both hairpin classes, propensity values greater than 1.5 are considered significant.

$\begin{array}{cccccccccc}\text { E-E- } \alpha_{R}-\alpha_{R}-\gamma_{R}-\alpha_{L}-E-E & S-2 & S-1 & T 1 & T 2 & T 3 & T 4 & S+1 & S+2 & \text { Residue } \\ & 0.03 & \mathbf{0 . 4 8} & 0.08 & 0.10 & 0.03 & 0.11 & 0.03 & 0.00 & \text { Asp } \\ & & \mathbf{7 . 1 1} & 0.51 & 0.68 & 0.17 & 0.78 & 0.17 & & \\ & 0.02 & \mathbf{0 . 2 1} & 0.05 & 0.03 & 0.12 & \mathbf{0 . 2 4} & 0.02 & 0.00 & \text { Asn } \\ & \mathbf{2 . 3 0} & 0.44 & 0.21 & 1.06 & \mathbf{2 . 8 4} & 0.14 & & \\ & 0.01 & 0.00 & \mathbf{0 . 1 6} & 0.03 & 0.00 & 0.00 & 0.00 & 0.03 & \text { Pro } \\ & & 0 & \mathbf{3 0} & 0.83 & 0 & 0 & 0 & & \\ & 0.00 & 0.01 & 0.01 & 0.04 & 0.02 & \mathbf{0 . 1 7} & 0.02 & 0.02 & \text { Gly } \\ & 0.18 & 0.18 & 1.00 & 0.46 & \mathbf{8 . 0 4} & 0.36 & & \\ & 0.04 & 0.00 & 0.06 & 0.04 & \mathbf{0 . 2 2} & 0.00 & \mathbf{0 . 1 8} & 0.05 & \text { Thr } \\ & 0.04 & 0.69 & 0.42 & \mathbf{3 . 7 1} & 0.04 & \mathbf{2 . 7 7} & & \\ & 0.02 & 0.04 & 0.07 & \mathbf{0 . 1 5} & \mathbf{0 . 1 5} & 0.05 & 0.06 & 0.04 & \text { Ser } \\ & 0.47 & 0.78 & \mathbf{1 . 9 4} & \mathbf{2 . 0 2} & 0.57 & 0.62 & & \\ & 0.01 & 0.04 & 0.01 & 0.00 & 0.01 & 0.02 & 0.01 & 0.03 & \text { His } \\ & 4.5 & 0.5 & 0.2 & 0.5 & 1.2 & 0.5 & & \\ & 0.02 & 0.01 & 0.11 & \mathbf{0 . 1 3} & 0.07 & 0.03 & 0.04 & 0.07 & \text { Ala } \\ & 0.12 & \mathbf{2 . 0 2} & \mathbf{2 . 6 3} & 1.12 & 0.37 & 0.51 & & \\ & 0.01 & 0.03 & 0.05 & \mathbf{0 . 1 5} & 0.08 & 0.05 & 0.09 & 0.03 & \text { Glu } \\ & 0.32 & 0.67 & \mathbf{2 . 5 3} & 1.01 & 0.61 & 1.32 & & \\ & 0.04 & 0.01 & 0.03 & 0.03 & 0.03 & 0.07 & 0.09 & 0.03 & \text { Arg } \\ & 0.18 & 0.60 & 0.72 & 0.60 & \mathbf{1 . 8 3} & \mathbf{2 . 7 7} & & \\ & 0.06 & 0.01 & 0.09 & 0.03 & 0.08 & 0.02 & 0.04 & \mathbf{0 . 1 7} & \text { Leu } \\ & 0.17 & \mathbf{2 . 3 7} & 0.67 & \mathbf{2 . 0 2} & 0.37 & 1.02 & & \\ & 0.04 & 0.01 & 0.11 & 0.09 & \mathbf{0 . 1 3} & \mathbf{0 . 1 8} & \mathbf{0 . 1 3} & 0.05 & \text { Lys } \\ & 0.11 & 1.04 & 0.80 & 1.20 & \mathbf{1 . 9 1} & 1.25 & & \end{array}$

The suggested loop sequences based on this statistical data are:

$\begin{array}{lcccccc}1^{\text {st }} \text { choice } & \text { Asp } & \text { Pro } & \text { Ala } & \text { Thr } & \text { Gly } & \text { Thr } \\ 2^{\text {nd }} \text { choice } & \text { Asn } & \text { Ala } & \text { Glu } & \text { Ser } & \text { Asn } & \text { Arg } \\ 3^{\text {rd }} \text { choice } & \text { (His) } & \text { (Leu) } & \text { Ser } & \text { Leu } & \text { Lys } & \text { Lys }\end{array}$




$\begin{array}{ccccccccccc}\text { E-b- } \alpha_{R}-\alpha_{R}-\gamma_{R}-\alpha_{L}-b-E & \text { S-2 } & \text { S-1 } & \text { T1 } & \text { T2 } & \text { T3 } & \text { T4 } & \text { S+1 } & \text { S+2 } & \text { Residue } \\ & 0.00 & \mathbf{0 . 3 1} & 0.06 & 0.08 & 0.05 & 0.03 & 0.05 & 0.00 & \text { Asp } \\ & 0 & \mathbf{5 . 5 9} & 0.63 & 0.81 & 0.45 & 0.29 & 0.45 & & \\ & 0.00 & \mathbf{0 . 2 1} & 0.00 & 0.08 & 0.05 & 0.03 & 0.00 & 0.03 & \text { Asn } \\ & & \mathbf{7 . 0 0} & 0.00 & \mathbf{1 . 3 2} & 0.71 & 0.45 & 0.00 & & \\ & 0.02 & 0.00 & \mathbf{0 . 2 3} & 0.02 & 0.03 & 0.00 & 0.00 & 0.05 & \text { Pro } \\ & & 0.00 & \mathbf{2 3 . 3} & 0.31 & 0.67 & 0.00 & 0.00 & & \\ & 0.00 & 0.00 & 0.02 & 0.02 & 0.00 & \mathbf{0 . 6 6} & 0.02 & 0.00 & \text { Gly } \\ & 0.00 & 0.12 & 0.12 & 0.00 & \mathbf{6 8 . 3} & 0.12 & & \\ & 0.03 & 0.02 & 0.05 & 0.10 & \mathbf{0 . 3 9} & 0.00 & 0.06 & 0.05 & \text { Thr } \\ & 0.14 & 0.43 & 0.94 & \mathbf{8 . 5 7} & 0.00 & 0.59 & & \\ & 0.03 & 0.05 & 0.05 & 0.08 & \mathbf{0 . 3 2} & 0.00 & 0.05 & 0.05 & \text { Ser } \\ & 0.48 & 0.48 & 0.86 & \mathbf{7 . 1 4} & 0.00 & 0.48 & & \\ & 0.06 & 0.06 & 0.02 & 0.03 & 0.02 & 0.02 & 0.03 & 0.00 & \text { His } \\ & \mathbf{2 . 8 6} & 0.50 & 1.11 & 0.50 & 0.50 & 1.11 & & \\ & 0.00 & 0.05 & 0.05 & 0.10 & 0.00 & 0.00 & 0.05 & 0.03 & \text { Ala } \\ & 1.25 & 1.25 & \mathbf{3 . 3 3} & 0.00 & 0.00 & 1.25 & & \\ & 0.03 & 0.05 & 0.03 & 0.11 & 0.02 & 0.05 & \mathbf{0 . 2 3} & 0.11 & \text { Glu } \\ & 0.56 & 0.36 & \mathbf{1 . 5 2} & 0.17 & 0.56 & \mathbf{4 . 3 8} & & \\ & 0.11 & 0.03 & 0.10 & 0.05 & 0.00 & 0.05 & \mathbf{0 . 1 5} & 0.08 & \text { Arg } \\ & 0.48 & \mathbf{1 . 7 6} & 0.75 & 0.00 & 0.75 & \mathbf{3 . 2 1} & & \\ & 0.08 & 0.05 & \mathbf{0 . 1 6} & 0.03 & 0.02 & 0.00 & 0.00 & 0.08 & \text { Leu } \\ & 1.15 & \mathbf{8 . 3 3} & 0.71 & 0.33 & 0.00 & 0.00 & & \\ & \mathbf{0 . 1 6} & 0.00 & 0.10 & 0.11 & 0.00 & 0.06 & \mathbf{0 . 1 1} & 0.08 & \text { Lys } \\ & 0.00 & 1.67 & \mathbf{2 . 0 6} & 0.00 & 1.00 & \mathbf{2 . 0 6} & & \end{array}$

The suggested loop sequences based on this statistical data are:

$\begin{array}{lllllll}1^{\text {st }} & \text { Asn } & \text { Pro } & \text { Ala } & \text { Thr } & \text { Gly } & \text { Glu } \\ 2^{\text {nd }} & \text { Asp } & \text { Leu } & \text { Glu } & \text { Ser } & \text { Gln } & \text { Arg } \\ 3^{\text {rd }} & \text { (His) } & \text { Arg } & \text { Lys } & & & \text { Lys }\end{array}$

Based on both sets of propensities and fractional occurrences, and our preference for positively charged or neutral sidechains over negatively charged ones (a solubility consideration), the following loop sequences have been selected for examination:

$\begin{array}{lllllll}1^{\text {st }} & \text { Asn } & \text { Pro } & \text { Ala } & \text { Thr } & \text { Gly } & \text { Lys } \\ 2^{\text {nd }} & \text { Asn } & \text { Pro } & \text { Ala } & \text { Thr } & \text { Gly } & \text { Thr } \\ 3^{\text {rd }} & \text { Asp } & \text { Pro } & \text { Ala } & \text { Thr } & \text { Gly } & \text { Arg } \\ 4^{\text {th }} & \text { His } & \text { Pro } & \text { Ala } & \text { Thr } & \text { Gly } & \text { Arg } \\ 5^{\text {th }} & \text { Asn } & \text { Ala } & \text { Ala } & \text { Thr } & \text { Gly } & \text { Arg } \\ 6^{\text {th }} & \text { Asn } & \text { Pro } & \text { Ala } & \text { Thr } & \text { Gly } & \text { Glu } \\ 7^{\text {th }} & \text { His } & \text { Asn } & \text { Pro } & \text { Ser } & \text { Gly } & \text { Asn } \\ 8^{\text {th }} & \text { Asp } & \text { Pro } & \text { Glu } & \text { Thr } & \text { Gly } & \text { Glu }\end{array}$

To date, the first four have yielded stable hairpin peptides (unpublished data). The $7^{\text {th }}$ loop sequence is based on statistics for hairpins with His in the S-1 position. The $8^{\text {th }}$ sequence will be used for hairpins with a large net negative charge. The present communication reports only the application of the $1^{\text {st }}$ loop sequence to GB1p analogs. 


\section{Chemical Shift Deviation Profiles and CD Melts for $\beta$ Hairpins}
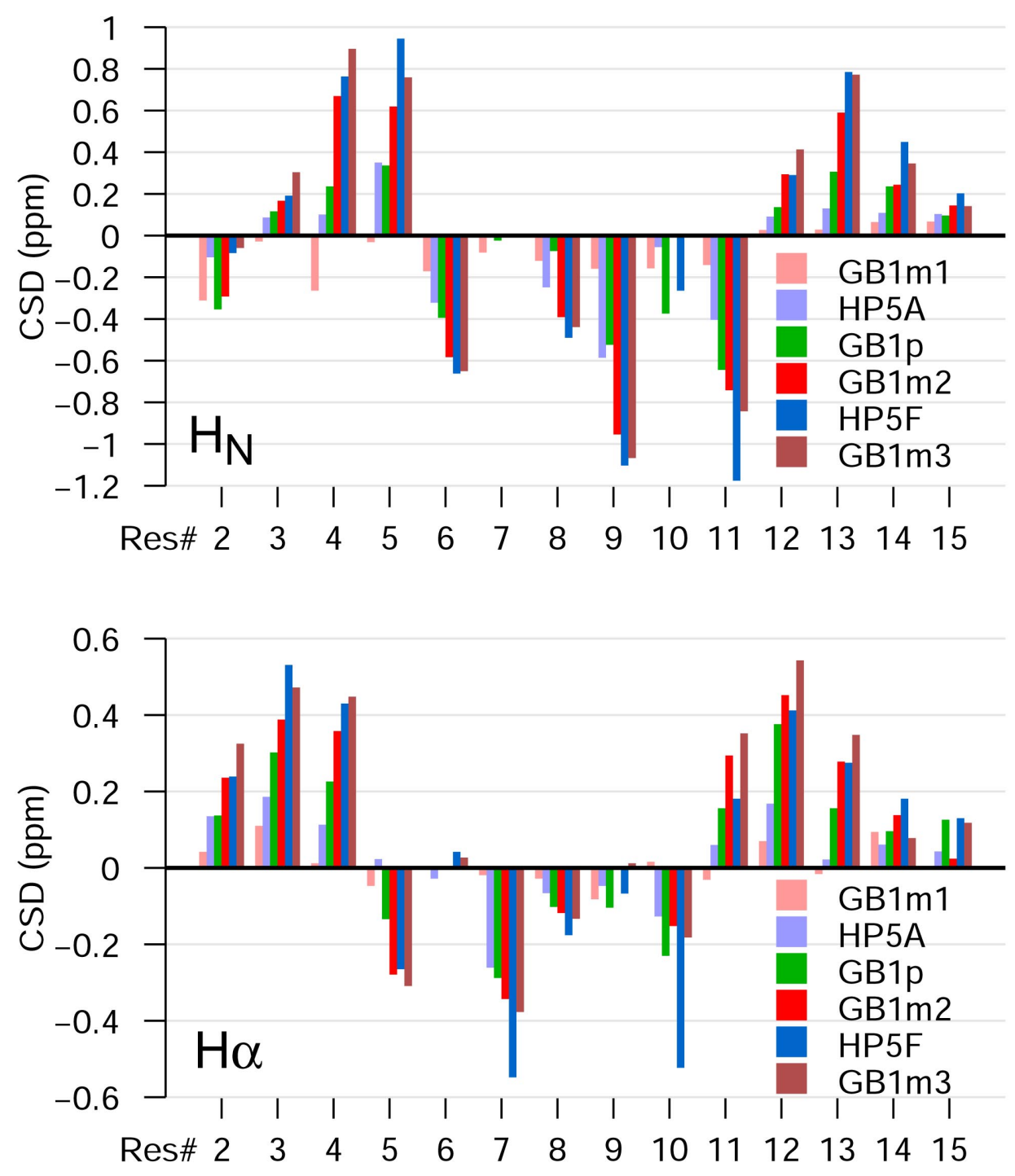

Figure 2S. Residue-by-residue comparisons of the $\mathrm{H}_{\mathrm{N}}$ (top panel) and $\mathrm{Ha}$ (lower panel) CSDs of peptides (in the order appearing) GB1m1 (F12A), HP5A, GB1m2, HP5F, and GB1m3. All data at $27^{\circ} \mathrm{C}$ except the literature data for GB1p which was reported at $5{ }^{\circ} \mathrm{C}$.

The steady increase in $|\mathrm{CSD}|$ values at positions $3 \alpha, 4 \alpha, 5 \alpha, 12 \alpha, 13 \alpha, 4 \mathrm{~N}, 6 \mathrm{~N}, 9 \mathrm{~N}, 13 \mathrm{~N}$, and $14 \mathrm{~N}$ provide the most direct measure of increasing folded fraction. HP5F displays a number of shifts that are not within the trend, presumably due to the differences in ring current and sidechain conformations associated with the rearrangement of aromatic residues within hydrophobic core. 
Even though the GB1m3 melting transition is rather broad, and the chemical shift changes per temperature increment are small, we have examined the reciprocal temperature plot method of Honda et al. ${ }^{2}$ The results appear in Figure 4S.
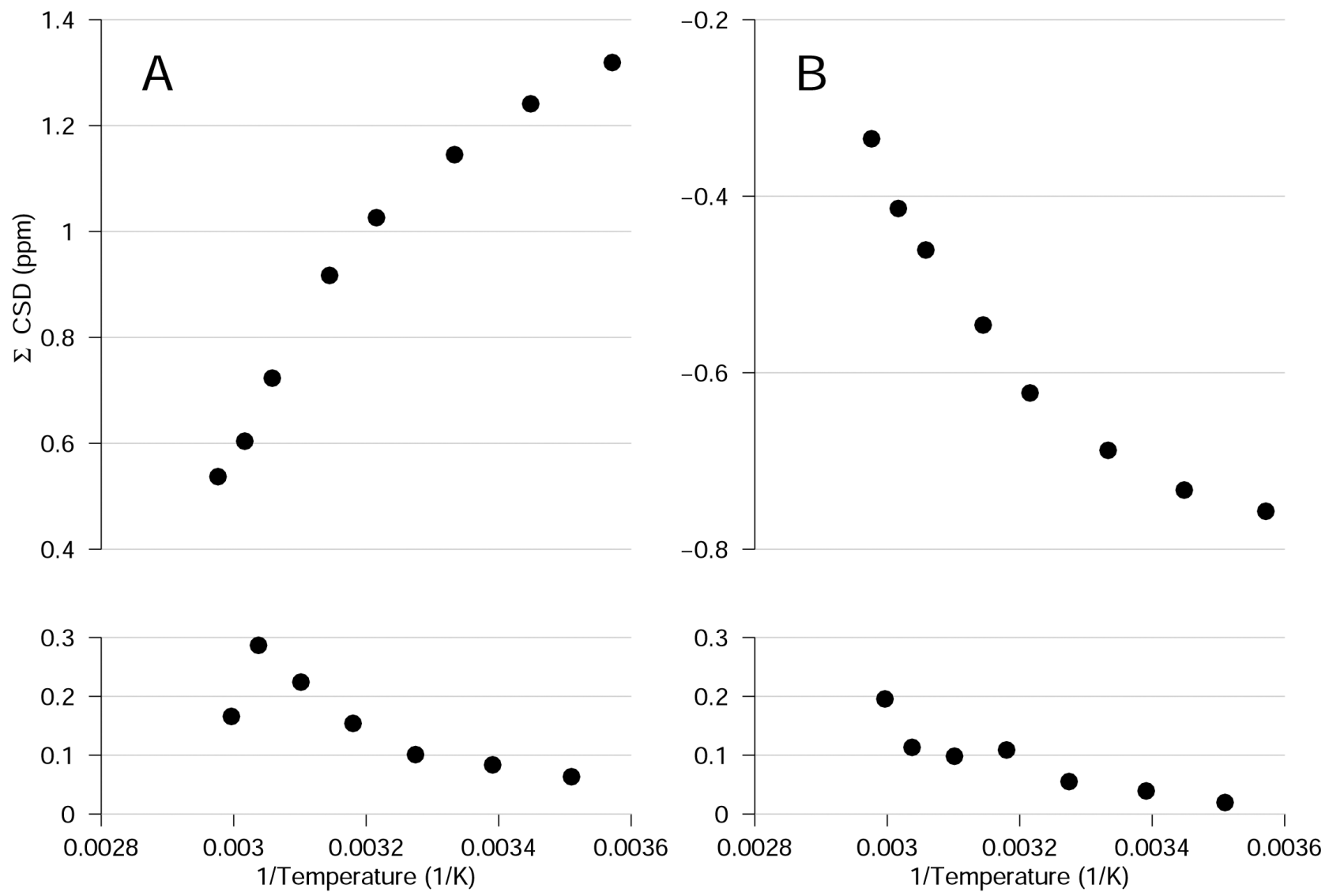

Figure 4S. Chemical shift (as sums of CSDs, no temperature corrections were applied to the random coil values) versus reciprocal temperature for key chemical shift measures of structuring in GB1m3. Panel A shows the sum of the CSDs for the most downfield-shifted $\mathrm{H} \alpha$ resonances at $\mathrm{H}$ bonded residues (res. 4, 11, and 13). Panel $\mathbf{B}$ is the comparable plot for the two largest upfield shifted $H \alpha$ sites (Y5 and P7). The lower traces are the absolute values $\left(\times 10^{-4}\right)$ of $d(C S D) / d(1 / T)$ vs $1 / \mathrm{T}$.

In the case of the strand $\mathrm{H} \alpha$ plot a maximum is observed in the derivative plot (at circa $57^{\circ} \mathrm{C}$ ); the 5,7-Ho plot is still increasing in slope at $62{ }^{\circ} \mathrm{C}$. Using the CSDs themselves, assuming a 0.96 folded fraction at $7{ }^{\circ} \mathrm{C}$, and defining $\mathrm{Tm}$ as the point at which the CSD falls to one half of the fully structured value, the Tm's are $56{ }^{\circ} \mathrm{C}$ and $60{ }^{\circ} \mathrm{C}$, respectively for panels $\mathbf{A}$ and $\mathbf{B}$. These are in agreement with the values derived from strand $\mathrm{NHs}\left(57^{\circ} \mathrm{C}\right)$, the $\mathrm{H \alpha}$ 's at residues 3 and $12\left(61{ }^{\circ} \mathrm{C}\right)$, and from the $\mathrm{CD}$ melt $\left(62^{\circ} \mathrm{C}\right)$. 
H $\alpha$ CSD melting data along the complete sequences of the reported peptides are in excellent agreement. The data for HP5F and HP5W4 are representative (Figure 8).
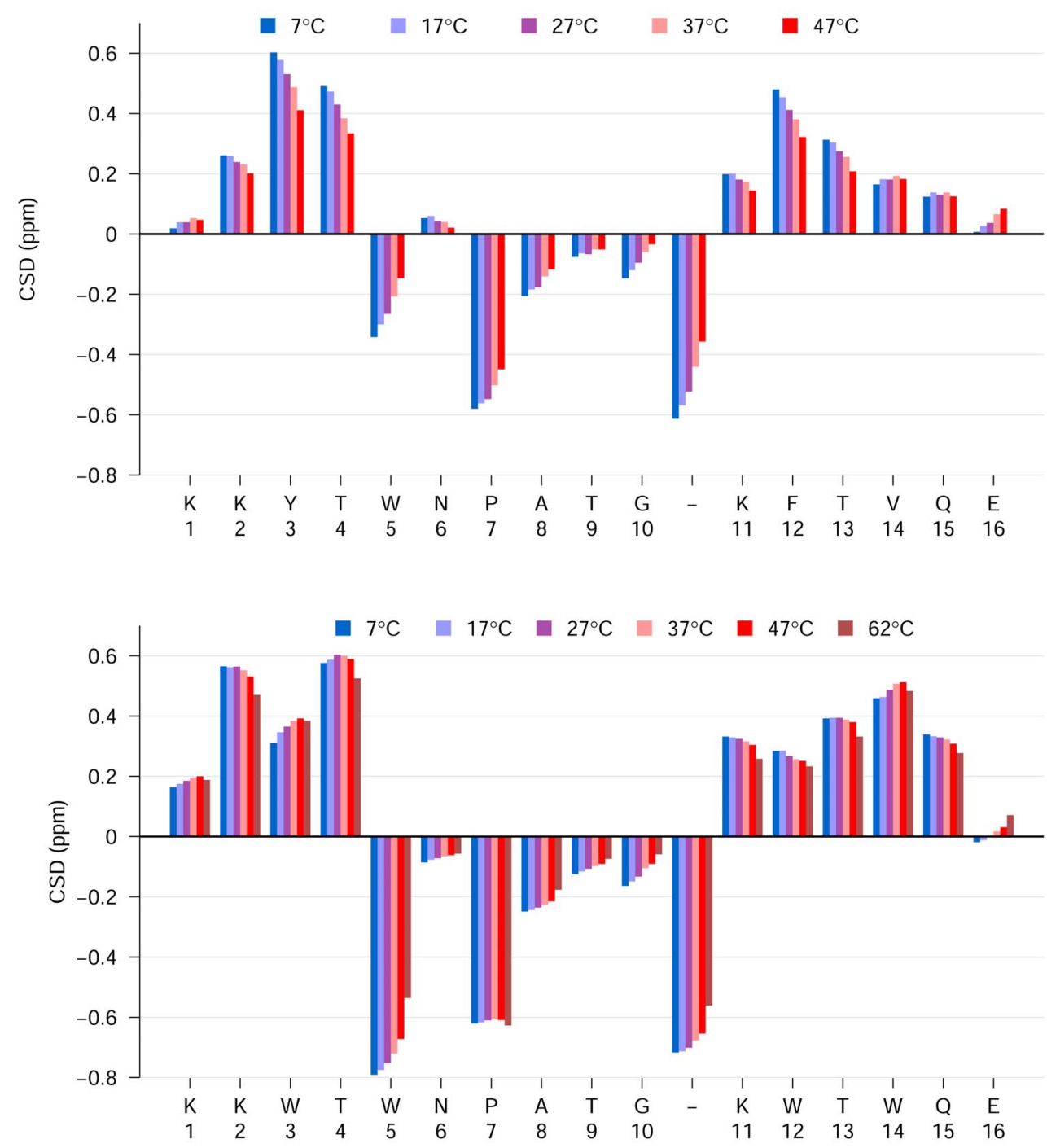

Figure 8. CSD versus temperature profile generated by our CSDb program: HP5F at $7-47^{\circ} \mathrm{C}$ (upper panel), HP5W4 at $7-62{ }^{\circ} \mathrm{C}$ (lower panel).

The data for HP5W4 was taken up to $62{ }^{\circ} \mathrm{C}$ since there was very little evidence of melting at the usual upper temperature limit for our studies. The observation that all CSDs, whether positive or negative, uniformly head toward zero indicates melting cooperativity and that the random coil and sequence context corrections employed are correct for this set of peptides.

The lack of chemical shift melting at Pro ${ }^{7}-\mathrm{H} \alpha$ in HP5W4 (and diminished melting at this site for HP5W and to some extent for all other systems examined) is notable. This likely reflects a smaller increase in the amplitude of $\phi / \psi$ variation on warming due to steric constraints of the proline ring. 
That the loop retains some structure even after the strand alignment melts out, cannot be dismissed. NMR shift melting comparisons within the GB1 mutant and HP5 series appear in Figure 9.
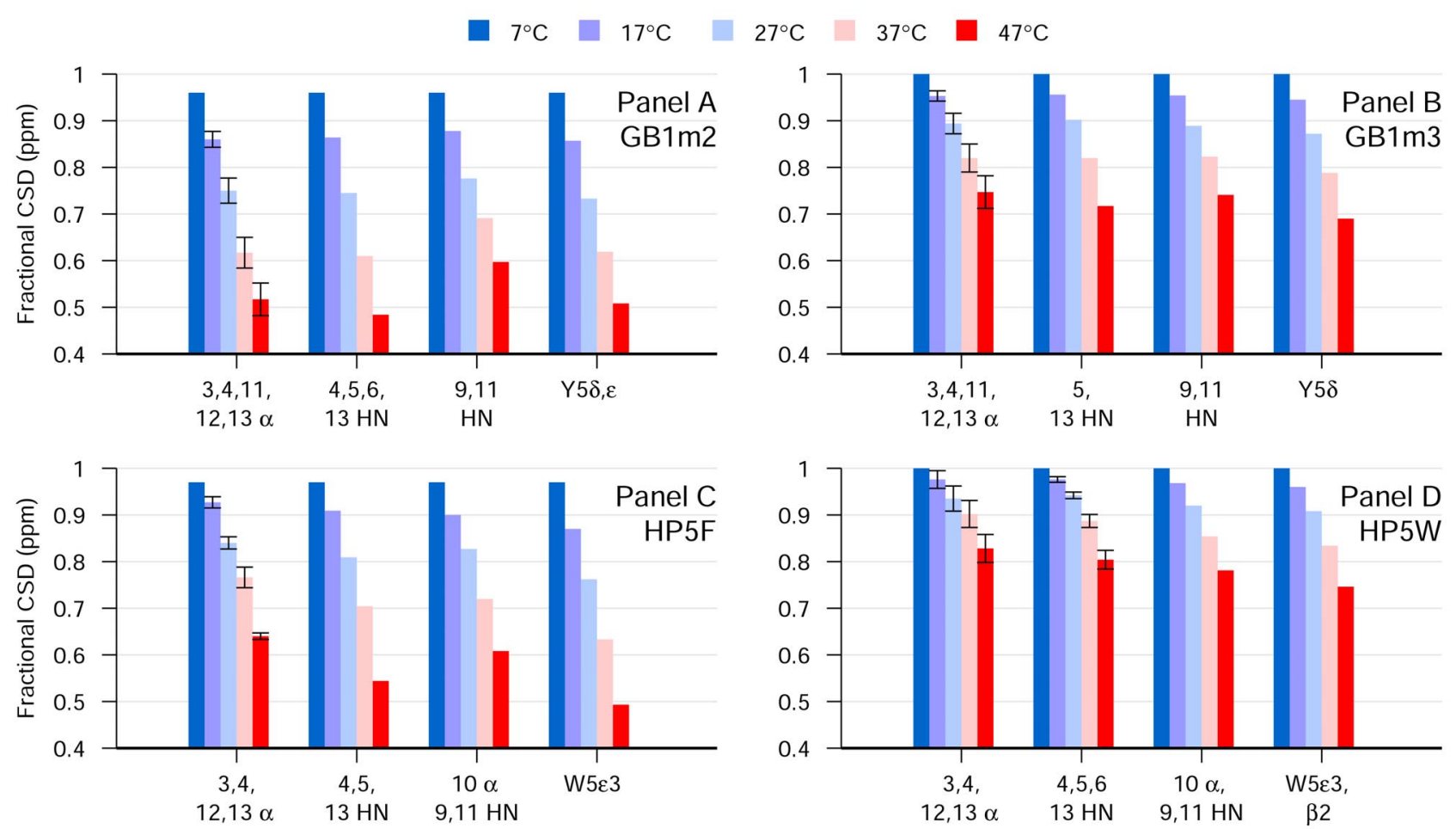

Figure 9. Folding cooperativity: comparisons of melting measures for GB1m2 (panel A), GB1m3 (panel B), HP5F (panel C), and HP5W4 (panel D). Measures 1) and 2) reflect strand alignment, measure 3) reflects loop stability, and measure 4) is purely due to aromatic ring current effects within the hydrophobic cluster. The specific shifts employed are indicated on the panels; when ' $10 \alpha$ ' is employed it is the upfield diastereomer of this $\mathrm{CH}_{2}$ group. In each comparison (GB1m2 vs GB1m3) and (HP5F vs HP5W4) the more folded species is the standard for the fractional CSD scale. The best estimates of the corresponding folded fractions at $280 \mathrm{~K}$ are 0.96 (GB1p) and 0.98 (HP5W). The further reduction in folded fraction for the less stable form is shown.

For HP5F, a slightly lower Tm is calculated based on the W5\&3 shift temperature dependence, which was excluded for the $\%$-folded calculation reported in Table 1 . This may indicate that sidechain $\chi$ variation in the hydrophobic core increases more rapidly with temperature than backbone motion; similar, though less dramatic, observations may be made for the aromatic sidechains of the other peptides shown in Figure 9. In the case of HP5W4 the temperature dependence of the chemical shift of the upfield $\operatorname{Trp}^{5}-\mathrm{H} \beta$ was also included. At the low temperature limit, the $\mathrm{C}_{\beta} \mathrm{H}_{2}$ group displays dramatically different shifts, 2.41 and $1.19 \mathrm{ppm}$. The upfield value represents a structure-induced shift of $1.97 \mathrm{ppm}$. 


\section{Chemical Shift Assignment Tables}

Tables 1S - 7S. Chemical shift assignments for peptides at $290 \mathrm{~K}$ in $20 \mathrm{mM} \mathrm{pH} 6$ phosphate buffer. Assignment values (in ppm) are relative to DSS (0 ppm).

Table 1S: GB1m1

\begin{tabular}{|l|l|l|l|l|}
\hline Residue & $\mathrm{HN}$ & $\mathrm{H} \alpha$ & $\mathrm{H} \beta, \mathrm{H} \beta^{\prime}$ & Others \\
\hline Gly1 & Exchange & $3.807,3.763$ & & \\
\hline Glu2 & 8.594 & 4.302 & 1.854 & $\gamma, \gamma^{\prime} 2.252,2.164$ \\
\hline Trp3 & 8.415 & 4.750 & $3.237,3.159$ & $\begin{array}{l}\varepsilon 110.057 ; \delta 17.157 ; \varepsilon 37.596 \\
\zeta 37.158 ; \eta 27.155 ; \zeta 27.458\end{array}$ \\
\hline Thr4 & 7.915 & 4.263 & 4.040 & $\gamma 1.062$ \\
\hline Tyr5 & 8.110 & 4.396 & $2.955,2.897$ & $\delta, \delta 7.092 ; \varepsilon, \varepsilon^{\prime} 6.813$ \\
\hline Asp6 & 8.277 & 4.553 & 2.771 & \\
\hline Asp7 & 8.262 & 4.580 & 2.740 & \\
\hline Ala8 & 8.224 & 4.310 & 1.418 & \\
\hline Thr9 & 8.028 & 4.267 & 4.234 & $\gamma 1.194$ \\
\hline Lys10 & 8.224 & 4.344 & $1.842,1.785$ & $\gamma, \gamma^{\prime} 1.433 ; \delta, \delta$ \\
& & & & $\mathcal{N}^{\prime} 1.656 ; \varepsilon^{\prime}, \varepsilon^{\prime} 3.304 ;$ \\
\hline Thr11 & 8.104 & 4.318 & 4.180 & $\gamma 1.196$ \\
\hline Ala12 & 8.410 & 4.416 & 1.392 & \\
\hline Thr13 & 8.267 & 4.333 & 4.142 & $\gamma 1.187$ \\
\hline Val14 & 8.305 & 4.221 & 2.057 & $\gamma, \gamma^{\prime} 0.924,0.906$ \\
\hline Thr15 & 8.362 & 4.354 & 4.182 & $\gamma 1.202$ \\
\hline Glu16 & 8.267 & 4.295 & $2.250,2.158$ & $\gamma, \gamma^{\prime} 2.409$ \\
\hline
\end{tabular}

Table 2S: GB1m2

\begin{tabular}{|c|c|c|c|c|}
\hline Residue & $\mathrm{HN}$ & $\mathrm{H} \alpha$ & $\mathrm{H} \beta, \mathrm{H} \beta^{\prime}$ & Others \\
\hline Gly1 & Exchange & na & & \\
\hline Glu2 & 8.591 & 4.535 & $2.003,1.887$ & $\gamma, \gamma^{\prime} 2.290,2.256$ \\
\hline Trp3 & 8.634 & 5.087 & $3.190,3.135$ & $\begin{array}{l}\varepsilon 110.077 ; \delta 17.193 ; \varepsilon 37.357 \\
\zeta 3 \quad 7.034 ; \eta 27.153 ; \zeta 27.358\end{array}$ \\
\hline Thr4 & 8.982 & 4.646 & 4.062 & $\gamma 1.175$ \\
\hline Tyr5 & 8.842 & 4.159 & $2.633,2.633$ & $\delta, \delta^{\prime} 6.275 ; \varepsilon, \varepsilon^{\prime} 6.488$ \\
\hline Asn6 & 7.703 & 4.971 & $3.127,2.532$ & $\gamma \mathrm{NH} 2$ n. a. \\
\hline Pro7 & ---------- & 4.007 & $2.376,2.376$ & $\gamma, \gamma^{\prime} 2.019 ; \delta, \delta \delta^{\prime} 3.808,3.743$ \\
\hline Ala8 & 7.927 & 4.219 & 1.427 & \\
\hline Thr9 & 7.162 & 4.381 & 4.246 & $\gamma 1.088$ \\
\hline Gly10 & 8.340 & $4.023,3.757$ & & \\
\hline Lys11 & 7.403 & 4.621 & $1.816,1.816$ & $\begin{array}{l}\gamma, \gamma^{\prime} 1.346,1.318 ; \delta, \delta \\
\varepsilon N H 37.586\end{array}$ \\
\hline Phe12 & 8.758 & 5.159 & $2.897,2.837$ & $\delta, \delta^{\prime} 7.283 ; \varepsilon, \varepsilon^{\prime} 7.283 ; \zeta 7.170$ \\
\hline Thr13 & 8.854 & 4.648 & 4.108 & $\gamma 1.163$ \\
\hline Val14 & 8.402 & 4.242 & 1.572 & $\gamma, \gamma^{\prime} 0.664,0.551$ \\
\hline Thr15 & 8.419 & 4.377 & 4.154 & $\gamma 1.189$ \\
\hline Glu16 & 8.253 & 4.210 & $2.109,1.913$ & $\gamma, \gamma^{\prime} 2.364$ \\
\hline
\end{tabular}


Table 3S: GB1m3

\begin{tabular}{|c|c|c|c|c|}
\hline Residue & $\mathrm{HN}$ & $\mathrm{H} \alpha$ & $\mathrm{H} \beta, \mathrm{H} \beta{ }^{\prime}$ & Others \\
\hline Lys1 & Exchange & 4.055 & $1.705,1.705$ & $\begin{array}{l}\gamma, \gamma^{\prime} 1.305,1.263 ; \delta, \delta ' 1.576 ; \varepsilon, \varepsilon^{\prime} 2.891 \\
\varepsilon N H 3 \text { na }\end{array}$ \\
\hline Lys2 & 8.690 & 4.583 & $1.801,1.719$ & $\begin{array}{l}\gamma, \gamma^{\prime} 1.403,1.357 ; \delta, \delta^{\prime} 1.661 ; \varepsilon, \varepsilon^{\prime} 2.949 \\
\varepsilon N H 3 \text {;.558 }\end{array}$ \\
\hline Trp3 & 8.759 & 5.149 & $3.144,3.144$ & $\begin{array}{l}\varepsilon 110.127 ; \delta 17.129 ; \varepsilon 37.389 \\
\zeta 37.054 ; \eta 27.182 ; \zeta 27.386\end{array}$ \\
\hline Thr4 & 9.145 & 4.705 & 4.064 & $\gamma 1.185$ \\
\hline Tyr5 & 8.932 & 4.129 & $2.635,2.635$ & $\delta, \delta 6.218 ; \varepsilon, \varepsilon^{\prime} 6.486$ \\
\hline Asn6 & 7.680 & 4.991 & $3.166,2.537$ & $\gamma \mathrm{NH} 2 \quad c a .7 .28$ \\
\hline Pro7 & --------- & 3.992 & $2.412,1.998$ & $\gamma, \gamma^{\prime} 2.081,2.046 ; \delta, \delta^{\prime} 3.825,3.784$ \\
\hline Ala8 & 7.902 & 4.212 & 1.430 & \\
\hline Thr9 & 7.096 & 4.394 & 4.247 & $\gamma 1.079$ \\
\hline Gly10 & 8.352 & $4.038,3.743$ & & \\
\hline Lys11 & 7.346 & 4.662 & $1.862,1.833$ & $\begin{array}{l}\gamma, \gamma^{\prime} 1.367,1.335 ; \delta, \delta^{\prime} 1.614,4.591 \\
\varepsilon, \varepsilon^{\prime} 2.998 ; \varepsilon N H 37.591\end{array}$ \\
\hline Phe12 & 8.865 & 5.226 & $2.955,2.888$ & $\delta, \delta 7.192 ; \varepsilon, \varepsilon^{\prime} 7.324 ; \zeta 7.256$ \\
\hline Thr13 & 8.995 & 4.698 & 4.129 & $\gamma 1.187$ \\
\hline Val14 & 8.451 & 4.124 & 1.508 & $\gamma, \gamma^{\prime} 0.624,0.416$ \\
\hline GIn15 & 8.537 & 4.439 & $2.027,1.860$ & 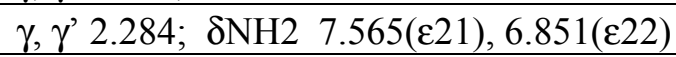 \\
\hline Glu16 & 8.337 & 4.083 & $2.065,1.882$ & $\gamma, \gamma^{\prime} 2.318$ \\
\hline
\end{tabular}

Table 4S: HP5A

\begin{tabular}{|c|c|c|c|c|}
\hline Residue & $\mathrm{HN}$ & $\mathrm{H} \alpha$ & $\mathrm{H} \beta, \mathrm{H} \beta^{\prime}$ & Others \\
\hline Lys 1 & Exchange & 3.996 & $1.810,1.810$ & $\gamma, \gamma^{\prime} 1.336 ; \delta, \delta^{\prime} 1.662 ; \varepsilon, \varepsilon^{\prime} 2.952$ \\
\hline Lys2 & 8.689 & 4.406 & $1.718,1.718$ & $\gamma, \gamma^{\prime} 1.346 ; \delta, \delta^{\prime} 1.684 ; \varepsilon, \varepsilon^{\prime} 2.950$ \\
\hline Tyr3 & 8.593 & 4.729 & $2.838,2.836$ & $\delta, \delta 7.041 ; \varepsilon, \varepsilon^{\prime} 6.772$ \\
\hline Thr4 & 8.318 & 4.380 & 4.061 & $\gamma 1.136$ \\
\hline Trp5 & 8.483 & 4.640 & $3.175,3.174$ & $\begin{array}{l}\varepsilon 110.17 ; \delta 1 ; \varepsilon 37.513 \\
\zeta 3 \quad 7.185 ; \eta 27.103 ; \zeta 27.431\end{array}$ \\
\hline Asn6 & 8.019 & 4.889 & $2.944,2.541$ & $\gamma \mathrm{NH} 27.525(\delta 21), 7.071(\delta 22)$ \\
\hline Pro7 & ---------- & 4.063 & $2.280,1.941$ & $\gamma, \gamma^{\prime} 1.959 ; \delta, \delta \prime 3.692,3.543$ \\
\hline Ala8 & 8.104 & 4.279 & 1.409 & \\
\hline Thr9 & 7.608 & 4.382 & 4.245 & $\gamma 1.139$ \\
\hline Gly10 & 8.305 & $3.974,3.807$ & & \\
\hline Lys11 & 7.835 & 4.382 & $1.781,1.781$ & $\begin{array}{l}\gamma, \gamma^{\prime} 1.368 ; \delta, \delta \prime 1.655 ; \varepsilon, \varepsilon^{\prime} 2.962 \\
\varepsilon N H 37.545\end{array}$ \\
\hline Ala12 & 8.504 & 4.533 & 1.299 & \\
\hline Thr13 & 8.361 & 4.377 & 4.124 & $\gamma 1.163$ \\
\hline Val14 & 8.310 & 4.143 & 1.994 & $\gamma, \gamma^{\prime} 0.867$ \\
\hline GIn15 & 8.536 & 4.364 & $2.050,1.923$ & 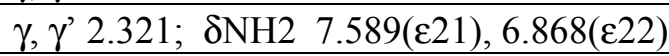 \\
\hline Glu16 & 8.298 & 4.208 & $2.112,1.919$ & $\gamma, \gamma^{\prime} 2.388$ \\
\hline
\end{tabular}


Table 5S: HP5F

\begin{tabular}{|c|c|c|c|c|}
\hline Residue & $\mathrm{HN}$ & $\mathrm{H} \alpha$ & $\mathrm{H} \beta, \mathrm{H} \beta^{\prime}$ & Others \\
\hline Lys1 & Exchange & 4.055 & 1.763 & $\gamma, \gamma 1.362 ; \delta, \delta^{\prime} 1.662 ; \varepsilon, \varepsilon^{\prime} 2.981 ; \varepsilon N H 37.574$ \\
\hline Lys2 & 8.705 & 4.527 & $1.777,1.715$ & $\begin{array}{l}\gamma, \gamma^{\prime} 1.378,1.326 ; \delta, \delta \prime 1.676 ; \varepsilon, \varepsilon^{\prime} 2.960 \\
\varepsilon N H 37.573\end{array}$ \\
\hline Tyr3 & 8.719 & 5.110 & $2.817,2.817$ & $\delta, \delta 6.941 ; \varepsilon, \varepsilon^{\prime} 6.709$ \\
\hline Thr4 & 9.045 & 4.735 & 4.081 & $\gamma 1.239$ \\
\hline Trp5 & 9.142 & 4.326 & $3.060,2.993$ & 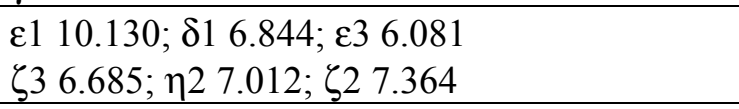 \\
\hline Asn6 & 7.625 & 4.973 & $3.119,2.478$ & $\gamma \mathrm{NH} 2 \quad 7.272(\delta 21), 7.22(\delta 22)$ \\
\hline Pro7 & --------- & 3.792 & $2.395,1.976$ & $\gamma, \gamma^{\prime} 2.057 ; \delta, \delta, 3.792,3.648$ \\
\hline Ala8 & 7.852 & 4.178 & 1.420 & \\
\hline Thr9 & 7.045 & 4.320 & 4.167 & $\gamma 1.038$ \\
\hline Gly10 & 8.060 & $3.812,3.363$ & & \\
\hline Lys11 & 6.948 & 4.491 & 1.780 & $\begin{array}{l}\gamma, \gamma^{\prime} 1.481,1.271 ; \delta, \delta \delta^{\prime} 1.676,1.579 ; \\
\varepsilon, \varepsilon^{\prime} 2.979 ; \varepsilon N H 37.573\end{array}$ \\
\hline Phe12 & 8.752 & 5.095 & $2.970,2.897$ & $\delta, \delta 7.280 ; \varepsilon, \varepsilon^{\prime} 7.412 ; \zeta 7.197$ \\
\hline Thr13 & 9.062 & 4.625 & 4.171 & $\gamma 1.193$ \\
\hline Val14 & 8.588 & 4.264 & 1.932 & $\gamma, \gamma^{\prime} 0.795$ (both) \\
\hline GIn15 & 8.625 & 4.470 & $2.054,1.895$ & 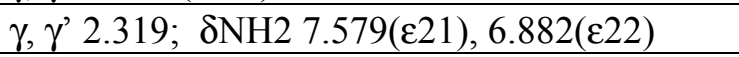 \\
\hline Glu16 & 8.436 & 4.169 & $2.117,1.939$ & $\gamma, \gamma^{\prime} 2.418$ \\
\hline
\end{tabular}

Table 6S: HP5W

\begin{tabular}{|c|c|c|c|c|}
\hline Residue & $\mathrm{HN}$ & $\mathrm{H} \alpha$ & $\mathrm{H} \beta, \mathrm{H} \beta^{\prime}$ & Others \\
\hline Lys1 & Exchange & 4.078 & $1.764,1.764$ & $\begin{array}{l}\gamma, \gamma^{\prime} 1.386 ; \delta, \delta \\
\varepsilon N H 37.583\end{array}$ \\
\hline Lys2 & 8.715 & 4.589 & $1.804,1.724$ & $\begin{array}{l}\gamma, \gamma^{\prime} 1.393,1.352 ; \delta, \delta \delta^{\prime} 1.682 ; \varepsilon, \varepsilon^{\prime} 2.973 \\
\varepsilon N H 37.583\end{array}$ \\
\hline Tyr3 & 8.771 & 5.305 & $3.060,2.836$ & $\delta, \delta 6.953 ; \varepsilon, \varepsilon^{\prime} 6.690$ \\
\hline Thr4 & 9.377 & 4.923 & 4.066 & $\gamma 1.232$ \\
\hline Trp5 & 9.003 & 4.098 & $2.639,1.766$ & $\begin{array}{l}\varepsilon 110.009 ; \delta 16.619 ; \varepsilon 35.519 \\
\zeta 36.513 ; \eta 26.945 ; \zeta 27.307\end{array}$ \\
\hline Asn6 & 7.415 & 4.928 & $3.066,2.425$ & $\gamma \mathrm{NH} 27.183(\delta 21), 7.245(\delta 22)$ \\
\hline Pro7 & ---------- & 3.717 & $2.401,1.986$ & $\gamma, \gamma^{\prime} 2.053 ; \delta, \delta, 3.798,3.658$ \\
\hline Ala8 & 7.787 & 4.150 & 1.407 & \\
\hline Thr9 & 6.886 & 4.280 & 4.140 & $\gamma 1.014$ \\
\hline Gly10 & 8.005 & $3.754,3.222$ & & \\
\hline Lys11 & 6.632 & 4.550 & $1.764,1.764$ & $\begin{array}{l}\gamma, \gamma^{\prime} 1.443,1.272 ; \delta, \delta \delta^{\prime} 1.690,1.583 \\
\varepsilon, \varepsilon^{\prime} 2.986 ; \varepsilon N H 37.583\end{array}$ \\
\hline Trp12 & 8.803 & 5.129 & $3.164,3.164$ & $\begin{array}{l}\varepsilon 110.231 ; \delta 17.512 ; \varepsilon 37.144 \\
\zeta 37.635 ; \eta 27.486 ; \zeta 27.239\end{array}$ \\
\hline Thr13 & 9.471 & 4.774 & 4.184 & $\gamma 1.210$ \\
\hline Val14 & 8.686 & 4.303 & 1.914 & $\gamma, \gamma^{\prime} 0.766$ (both) \\
\hline GIn15 & 8.664 & 4.501 & $2.057,1.888$ & $\gamma, \gamma^{\prime} 2.331 ; \delta \mathrm{NH} 2 \quad 7.598(\varepsilon 21), 6.885(\varepsilon 22)$ \\
\hline Glu16 & 8.446 & 4.147 & $2.115,1.931$ & $\gamma, \gamma^{\prime} 2.413$ \\
\hline
\end{tabular}


Table 7S:HP5W4

\begin{tabular}{|c|c|c|c|c|}
\hline Residue & $\mathrm{HN}$ & $\mathrm{H} \alpha$ & $\mathrm{H} \beta, \mathrm{H} \beta^{\prime}$ & Others \\
\hline Lys1 & Exchange & 4.174 & $1.704,1.704$ & $\begin{array}{l}\gamma, \gamma^{\prime} 1.268 ; \delta, \delta \\
\varepsilon N H 37.293\end{array}$ \\
\hline Lys2 & 8.689 & 4.807 & $1.856,1.794$ & $\begin{array}{l}\gamma, \gamma^{\prime} 1.401 ; \delta, \delta \delta^{\prime} 1.715 ; \varepsilon, \varepsilon^{\prime} 3.033 \\
\varepsilon N H 37.611\end{array}$ \\
\hline Trp3 & 9.134 & 4.981 & $3.230,2.580$ & $\begin{array}{l}\varepsilon 19.504 ; \delta 16.940 ; \varepsilon 36.354 \\
\zeta 36.759 ; \eta 27.085 ; \zeta 27.1456\end{array}$ \\
\hline Thr4 & 9.456 & 4.801 & 3.939 & $\gamma 1.149$ \\
\hline Trp5 & 8.675 & 3.799 & $2.428,1.240$ & $\begin{array}{l}\varepsilon 19.916 ; \delta 16.437 ; \varepsilon 35.604 \\
\zeta 36.619 ; \eta 26.970 ; \zeta 27.314\end{array}$ \\
\hline Asn6 & 7.269 & 4.834 & $3.034,2.361$ & $\gamma \mathrm{NH} 2 \quad 7.212(\delta 21), 7.179(\delta 22)$ \\
\hline Pro7 & ---------- & 3.586 & $2.340,1.918$ & $\gamma, \gamma^{\prime} 1.994 ; \delta, \delta \prime 3.726,3.572$ \\
\hline Ala8 & 7.729 & 4.117 & 1.380 & \\
\hline Thr9 & 6.817 & 4.267 & 4.128 & $\gamma 0.994$ \\
\hline Gly10 & 7.974 & $3.766,3.201$ & & \\
\hline Lys11 & 6.647 & 4.600 & $1.732,1.732$ & $\begin{array}{l}\gamma, \gamma^{\prime} 1.419,1.263 ; \delta, \delta^{\prime} 1.677,1.580 \\
\varepsilon, \varepsilon^{\prime} 2.988 ; \varepsilon N H 37.593\end{array}$ \\
\hline Trp12 & 8.634 & 4.920 & $2.762,2.288$ & $\begin{array}{l}\varepsilon 110.093 ; \delta 17.421 ; \varepsilon 36.909 \\
\zeta 37.094 ; \eta 27.178 ; \zeta 27.178\end{array}$ \\
\hline Thr13 & 8.966 & 4.606 & 4.131 & $\gamma 1.100$ \\
\hline Trp14 & 8.645 & 5.043 & $2.835,2.729$ & $\begin{array}{l}\varepsilon 110.034 ; \delta 17.229 ; \varepsilon 36.783 \\
\zeta 36.972 ; \eta 27.106 ; \zeta 27.236\end{array}$ \\
\hline GIn15 & 8.795 & 4.577 & $2.171,1.898$ & $\gamma, \gamma^{\prime} 2.283 ; \delta \mathrm{NH} 2 \quad 7.561(\varepsilon 21), 6.838(\varepsilon 22)$ \\
\hline Glu16 & 8.529 & 4.064 & $2.090,1.996$ & $\gamma, \gamma, 2.467$ \\
\hline
\end{tabular}

\section{References}

(1) Burke, D. F.; Deane, C. M.; Blundell, T. L. Bioinformatics 2000, 16, 513-519.

(2) Honda, S.; Kobayashi, N.; Munekata, E. J. Mol. Biol. 2000, 269-278. 